\title{
Spitze Zunge gegen Luther
}

\author{
Judith Keßler
}

Jeder Mensch hat einen freien Willen. So jedenfalls lautet das Fazit eines Gedichtes mit dem Titel So sijn dees woorden te vergeefs gesproken, auf Deutsch: Dann wurden diese Worte umsonst gesprochen, aus der Feder von Anna Bijns, einer streng katholischen niederländischen Dichterin (*1493 in Antwerpen; $\nmid 1575$ ebenda), die zeitgleich zu Martin Luthers ersten reformatorischen Ansätzen in Wittenberg im damals süd-niederländischen Antwerpen lebte und arbeitete. Bijns, die sehr an den aktuellen theologischen Diskussionen interessiert war und dank enger Kontakte zum örtlichen Franziskanerkloster wohl auch immer dementsprechend auf dem neuesten Stand war, wurde insbesondere für ihre angriffslustigen Gedichte gegen Luther bekannt, die seit 1527 in zahlreichen Handschriften und Drucken überliefert sind. Sie scheute nicht davor zurück, sich auch in komplexe Themen einzuarbeiten und ihre Meinung $\mathrm{zu}$ wichtigen Glaubensfragen zu verteidigen, wobei sie immer der Lehre der katholischen Kirche treu blieb und diese mit ihren Gedichten vor reformatorischen „Angriffen“ zu schützen versuchte. ${ }^{1}$

Das Gedicht über den freien Willen ist eines jener Gedichte, in denen Bijns dezidiert in einer theologischen Diskussion Stellung bezieht. In diesem Fall berührt sie einen zentralen Punkt christlichen Glaubens, nämlich die Frage, inwieweit der Mensch für seine Taten verantwortlich ist, inwieweit also Gott ihm die Möglichkeit gegeben hat, selbst über Gut und Böse zu entscheiden. Die Diskussion wird in den zwanziger Jahren des sechzehnten Jahrhunderts hauptsächlich zwischen Martin Luther und Desiderius Erasmus geführt, die ja beide für Reformen in der Kirche eintreten, wenn auch in unterschiedlich starker Form. ${ }^{2}$ Luther vertritt kurz zusammengefasst den Standpunkt, dass der Mensch keinen freien Willen habe, denn ein solcher freier Wille würde Gottes Allmacht Grenzen setzen. Das jedoch ist nach Luthers Überzeugung unmöglich, da die Allmacht sich gerade durch ihre Unbeschränktheit definiere. Also kann der Mensch auch nicht zum Beispiel durch den Kauf von Ablässen eine

1 Zur Biographie von Anna Bijns:Van den Branden (1911). Umfassend zu Leben und Werk: Roose (1963); Pleij (2007), Pleij (2011), Keßler (2013). Textausgaben: Keßler und Oosterman (2007a, b, c); Bogaers und Van Helten (1875); Jonckbloet und Van Helten (1886); Soens (1902).

2 Allgemein zur Diskussion um den freien Willen: Akerboom (1995). 
vorzeitige Beendigung etwaiger göttlicher Bestrafung erzwingen, denn Gott lässt sich nicht durch menschliches Handeln zwingen. Gott rettet den Menschen einzig, weil er barmherzig ist und weil er es will. Die Grundlage für diese Diskussion von Luthers Seite aus leitet sich aus seinen 95 Thesen zu Buße und Ablass aus dem Jahr 1517 ab, die $15^{20}$ in der Heidelberger Disputation noch weiter präzisiert werden sollten. ${ }^{3}$

Im Gegensatz dazu gesteht Erasmus dem Menschen ein gewisses Maß an freiem Willen zu, um über seine Handlungen bestimmen zu können. Das Böse, so argumentiert er, ist ja etwas zutiefst Menschliches und kann deshalb nicht von Gott kommen. Denn Gott ist zwar allmächtig, wie Luther sagt, darüber hinaus aber auch, anders als der Mensch, ganz und gar gut. Gott kann nichts Böses tun. Also kann nur der Mensch für das Böse verantwortlich sein, und zwar, indem er sich selbst entscheidet, ob er etwas Gutes oder Böses tun will. ${ }^{4}$ Somit ist der Mensch auch in der Lage, seine Sünden bis zu einem gewissen Grad wieder gutzumachen, bzw. dafür Buße zu tun und seine Schuld zu vermindern - und genau das gesteht Luther dem Menschen nicht zu.

Luther und Erasmus tragen ihre Diskussion insbesondere in zwei Schriften aus: Erasmus' De libero arbitrio diatribe sive collatio und Luthers Reaktion darauf, sein Traktat De servo arbitrio. ${ }^{5}$ Luthers harsche Antwort, in der er mit Erasmus ins Gericht geht, wird nicht nur wegen der unterschiedlichen theologischen Standpunkte, sondern auch wegen des rauen Tones, den Luther verwendet, zum Bruch zwischen beiden führen, obwohl sie vordem den gegenseitigen Reformansätzen durchaus positiv gegenüber gestanden haben.

3 Der Zusammenhang zwischen Luthers Thesen, der Heidelberger Disputation und Luthers Standpunkt zum freien Willen wird u.a. erläutert sowohl in Akerboom (1995), 121-123, als auch in Wenz' Vorwort zur Ausgabe von Erasmus' De libero arbitrio (Wenz (1998) 5). Zu Luthers Auffassung zur Allmacht Gottes und der daraus folgenden Unfreiheit des menschlichen Willens s. Lohse (2006) 110-112 und 121; Folkers (2006) 300; Bayer (2003) 170-171. Sowohl Folkers als auch Bayer leiten Luthers Auffassung ab aus dem, was er am Ende seines Traktats De servo arbitrio sagt: „Nun aber, da Gott mein Heil außerhalb meines Willens in seinen Willen aufgenommen hat, und nicht durch mein Werk oder Lauf, sondern durch seine Gnade und Barmherzigkeit versprochen hat, mich zu retten, so bin ich sicher und gewiß, daß jener treu sei und mich nicht belügt" (Folkers (2006) 300). Der freie Wille, so zeigt Folkers in Übereinstimmung mit Lohse weiterhin, bezieht sich für den Menschen ausschließlich auf Dinge, die unter ihm sind, und über die Gott ihn laut Genesis 1, 26-29 explizit als Herrscher angestellt hat (Folkers (2006) 290). Luther betont außerdem, „dass Gottes Vorherbestimmung und Erwählung, nicht aber die Gerechtigkeit des menschlichen Willens der Grund des Heils ist“ (Lohse (2006) 117). Vgl. hierzu auch Bayer (2003) 168-171 und Akerboom (1995) 131 und 270271.

4 Erasmus' Haltung wird erläutert in Bayer (2003) 170-173 und in Lohse (2006) 82-83. Außerdem: Wenz (1998) 5-7.

5 Erasmus (1524); Luther (1526). 
Diese Skizze der Diskussion soll zur Einführung in das Thema der Streitfrage genügen. Bevor ich nun auf Bijns' Gedicht selbst weiter eingehe und auf ihre Haltung in der Debatte, die der von Erasmus sehr nahe kommt, will ich kurz den literarischen Kontext umreißen, der für Bijns bestimmend ist. Zunächst will ich also die Frage nach ihr selbst als Autorin, nach ihrem Werk und nach ihrer bevorzugten Textgattung beantworten. Dies soll uns zu der zentralen Frage hinführen, warum gerade diese Gattung ihr für dieses Thema als besonders geeignet erschienen sein muss.

\section{Anna Bijns: Leben, Werk}

Die niederländische Dichterin Anna Bijns ist der deutschsprachigen Forschung zur Lutherrezeption im frühen sechzehnten Jahrhundert noch weitgehend unbekannt. ${ }^{6}$ Dies ist einerseits wegen der - sicherlich vorhandenen - Sprachbarriere erklärlich, andererseits aber verwunderlich, da Luther selbst insbesondere in den ersten Jahren nach dem Thesenanschlag gerade die Ereignisse in den Niederlanden mit großem Interesse verfolgte. Immerhin waren sie eines der ersten Länder, in das einige seine Ordensbrüder seine Vorstellung vom wahren christlichen Glauben vermittelten. Der wohl bekannteste Vertreter des Augustinerordens war Jacobus Praepositus, der schon seit 1519 in Antwerpen, dieser größten und wichtigsten der niederländischen Handelsstädte, als Prediger tätig war. Als Prior des dortigen Augustinerklosters führte er einen regen Briefwechsel mit Luther, doch nur wenig später zwangen ihn die Umstände, die es der neuen Lehre im von alters her katholischen Flandern sichtlich schwer machten, seinen Wohnsitz ins norddeutsche Bremen zu verlagern. ${ }^{7}$

Der (süd)niederländische Katholizismus wehrte sich in dieser Anfangsphase mit Händen und Füßen gegen die neue Lehre. Eine wichtige Rolle im Widerstand, auch später noch, spielte der bereits erwähnte Orden der Franziskaner,

6 Die einzige mir bekannte deutschsprachige Publikation zu Bijns ist bisher Schneiderwirth (1933).

7 Zu Praepositus:Janssen (1862). In diesem Zusammenhang sei auch an Praepositus' Mitbrüder Johannes van Esschen und Hendrik Vos erinnert, die 1523 wegen der Verkündigung ketzerischer Lehren vom Magistrat der Stadt zum Tod auf dem Scheiterhaufen verurteilt wurden. Das Los der beiden inspirierte Luther noch im gleichen Jahr zum Verfassen seines Sendbriefs an die Christen im Niederland und zum Schreiben eines Liedes mit dem Titel Ein neues Lied wir heben an. Letzteres sollte sich als medialer Geniestreich erweisen: frei von jeder Zensur und damit unbehelligt von katholischer Kontrolle, konnte die Lobeshymne auf den Märtyrertod der beiden Mönche in protestantischen Kreisen kursieren und die Brüder und Schwestern im Glauben zur Standhaftigkeit ermutigen; vgl. hierzu Akerboom und Gielis (2005). 
der wie die Augustiner ebenfalls in Antwerpen vertreten war. ${ }^{8}$ Im Gegensatz zu ihnen waren die Franziskaner keineswegs reformgesinnt, sondern predigten gegen Luther. Daneben wussten sie sich aber auch eines weiteren, sehr effektiven Mittels zu bedienen: sie spornten katholische Autoren an, volkssprachliche literarische Werke mit starker anti-protestantischer Tendenz zu schreiben, die vor allem von Laien gelesen werden sollten. Ganz besonders tat sich dabei Matthias Weynsen hervor, der umtriebige Prediger, der in den zwanziger Jahren des sechzehnten Jahrhundertes im Rheinland unterwegs war, gleichzeitig aber auch als Provinzial den Antwerpener Franziskanern vorstand und daher die Initialzündung der Verbreitung lutherischer Lehren in den Niederlanden in eben jener Stadt hautnah mitbekommen hatte. Er soll gleich mehrere Autoren zum Verfassen katholischen Propagandamaterials angestellt haben. ${ }^{9}$ Eine aber, die er erst gar nicht erst von der Wichtigkeit anti-lutherischer Texte überzeugen musste, war Bijns.

Anna Bijns verdiente ihren Lebensunterhalt als Lehrerin, beschäftigte sich aber in ihrer freien Zeit mit dem Schreiben von Gedichten. Sie hatte eine Vorliebe für das zu der Zeit populäre Genre des refrein, eine Weiterentwicklung der französischen Ballade, wie es von den bürgerlichen Dichtgenossenschaften der rederijkers mit Vorliebe verwendet wurde. Rederijkerskammern, wie diese Vereinigungen genannt wurden, gab es vor allem in den südlichen Niederlanden in nahezu jedem Dorf und jeder Stadt. In den Zusammenkünften widmete man sich vor allem der Produktion von Literatur, wobei insbesondere Poesie und Drama eine wichtige Rolle spielten. Da die rederijkers sich gerne in ihren literarischen Fähigkeiten maßen, hielten sie kammerinterne, aber auch lokale und große, mehrtägige interlokale Wettkämpfe ab, in denen mit einem besonders guten Theaterstück zu einem vorab festgesetzten Thema ein Preis gewonnen werden konnte. Diese Wettkämpfe entwickelten sich aus Schützenfesten heraus und bedeuteten für die Stadt, in der sie abgehalten wurden, einen enorm Zugewinn an Prestige. ${ }^{10}$

8 Über die Geschichte der Franziskaner allgemein: Robson (2006); Roest (2000). Insbesondere zu den Antwerpener Franziskanern (wenn auch ideologisch gefärbt): Schlager (1904); Schlager (1909).

9 Diese Meinung vertritt Herman Pleij, der es als erwiesen ansieht, dass Weynsen auch Bijns zum Verfassen von Literatur gegen Luther angespornt hätte; vgl. hierzu Pleij (2000) 203-207. Dazu auch: Roose (1963) 46-49. Zur Person von Weynsen: Bautz (2008) Sp. 996997 .

$10 \mathrm{Zu}$ den rederijkers gibt es eine Fülle niederländischsprachiger Literatur. Für grundlegende Informationen zu den Kammern und zur rederijkers-Literatur sind die folgenden Ausgaben zu empfehlen: Pleij (2007); Ramakers (2003); Oosterman und Ramakers (2001). Speziell zu verschiedenen Kammern: Van Bruaene (2008); Van Bruaene (2004); Van Dixhoorn 
Neben dem Theater war auch die Poesie für die rederijkers eine wichtige literarische Ausdrucksform, und das gilt ganz besonders für das refrein. Ein refrein besteht aus mindestens vier Strophen, von denen die letzte immer einem prince („Prinz") gewidmet ist. ${ }^{11}$ Am Ende einer jeden Strophe wird eine Refrainzeile wiederholt, die sogenannte stokregel, die den Inhalt der Strophe sinnspruchartig zusammenfasst. Die Qualität eines refreins lässt sich nach zeitgenössischer Vorstellung vor allem an der Komplexität des Reimschemas messen und an der Art und Weise, wie mit Sprache und Stilmitteln gespielt wird. Dabei gilt: je komplizierter die Reimformen, desto besser ist das refrein; eine Auffassung, bei der mitunter die Form wichtiger zu sein schien als der Inhalt, was den rederijkers vor allem in der Forschung des neunzehnten Jahrhunderts den Ruf einbrachte, der Epideiktik verfallen zu sein. ${ }^{12}$

Anna Bijns beherrschte das Fach des refreins genauso gut wie ihre männlichen rederijkers-Kollegen, zu deren Kammern sie als Frau jedoch keinen Zutritt hatte. Im Gegensatz zu einigen von ihnen wusste sie jedoch immer, die Balance zwischen Form und Inhalt zu halten: ihre Gedichte entsprechen formal höchsten rederijkers-Ansprüchen, kommen aber keineswegs als inhaltsleere Phrasen daher. Im Gegenteil: Bijns hatte, als orthodoxe Katholikin, eine ausgeprägte Meinung zu Luther und seiner neuen Lehre, wie sie sie auch in dem Gedicht zum freien Willen vertritt.

Für die Franziskaner waren Bijns' Gedichte ein Glücksgriff. Wie gesagt, spornten sie normalerweise katholische Autoren an um gegen Luther zu schreiben; in Bijns' Fall war das nicht nötig. Sie produzierte von sich aus, um Luther die Stirn zu bieten. Damit ihr Werk von einem größeren Publikum gelesen werden konnte, mussten die Franziskaner, allen voran Matthias Weynsen, ihre Gedichte nur noch bündeln und als Gedichtband drucken lassen. So kam es, dass neben den umfangreichen Handschriften A (Brüssel, KB, 19547) aus 15421545 und B (Gent, UB, 2166) aus 1529, beide mit jeweils mehr als hundert refreinen von Bijns, auch drei gedruckte Gedichtbände erschienen.

(2004); Moser (2001). Speziell zum rederijkers-Drama: Waite (2003); Hummelen (1958). Die einzige überlieferte zeitgenössische Poetik ist Matthijs de Casteleins De const van rethoriken (herausgegeben von Geirnaert (1986)).

11 Ursprünglich galt diese Widmung dem Vorsitzenden der Kammer, der prince genannt wurde. Im Laufe der Zeit konnten zum Beispiel in religiösen Gedichten Gott oder Christus gemeint sein, oder auch, in anderen Fällen, eine nicht weiter zu identifizierende Person. Auch die weibliche Form princersse („Prinzessin“) kommt sowohl in religiösen Gedichten (für Maria) als auch in Liebesgedichten (für die Geliebte) regelmäßig vor.

12 Zum refrein sind die folgenden Studien zu empfehlen: Coigneau (1980-1983); hier ist auch eine gute Definition des Genres zu finden (Coigneau (1980-1983) Bd. 1, 9-13). Außerdem: Van Elslander (1953). 
Der erste gedruckte Gedichtband, von dem man annimmt, dass Weynsen bei der Publikation geholfen hat, erschien 1528 unter dem Titel Schoon ende suverlijc boecxken inhoudende veel constige refereinen („Schönes und reines Büchlein, viele kunstvolle refreinen enthaltend"). ${ }^{13}$ Er enthält 23 refreinen, von denen viele explizit Luthers Lehre als ketzerisch und teuflisch anprangern; oftmals deutet Bijns mit einem bekannten katholischen Bild über die Ketzer an, dass Luther und seine Anhänger Abgesandte des Teufels oder gar „irdische Teufel“ seien. ${ }^{14}$ Das Gedicht über den freien Willen ist das zwanzigste in dieser Sammlung.

Um 1548 erscheint der zweite Band, das Tweede boeck vol schoone ende constighe refereynen (das "Zweite Buch voll schöner und kunstvoller refreinen“). Auch dieser Band enthält 23 ebenfalls vor allem gegen Luther gerichtete refreinen von Bijns, aber darüber hinaus auch ein refrein eines Priesters und rederijkers, des Genters Stevijn van den Gheenste, der Bijns für ihren tapferen Kampf gegen Luther lobt.

Der dritte und letzte Band erscheint 1567 unter dem Titel Seer scoon ende suyver boeck, verclarende die mogentheyt Gods, ende Christus ghenade, over die sondighe menschen („Sehr schönes und reines Buch, erklärend die Macht Gottes, und Christi Gnade, über die sündigen Menschen“). Die refreinen, die hier gesammelt wurden, 70 an der Zahl, kennzeichnen sich vor allem durch ihren religiösen und devoten Inhalt. Luthers Lehre ist (fast) kein Thema mehr, und die Gedichte sind auch weit weniger angriffslustig und polemisch als die in den ersten beiden Bänden. Immerhin hatte sich ein Jahr vorher der Bildersturm zu Antwerpen ereignet; die katholische Bevölkerung stand noch unter dem Schock der Ereignisse, die die katholische Regierung in ihren Grundfesten erschüttert hatte. ${ }^{15}$ Wie viele andere katholische Einrichtungen auch, hatte das Franziskanerkloster besonders zu leiden: Es war in den Wirren des Bildersturmes abgebrannt. ${ }^{16}$ Und so erfüllte der Sammelband mit Bijns' refrei-

13 Für die Textausgaben der drei Bände und der beiden Handschriften s. Fußnote 1.

14 So lautet zum Beispiel der Titel des siebzehnten refreins desselben Gedichtbandes Tsijn eertsce duvels die de menscen quellen („Sie sind irdische Teufel, die die Menschen quälen“). Dem refrein zufolge mit dem Titel Dit comt meest al tsamen uut Luthers doctrijne („Das kommt alles zusammen von Luthers Lehre“), Nummer 15 aus der Sammlung, ist Luther sogar vom Teufel besessen: De prince der duvelen / is ontbonden / En hi heeft sijn ingelen uut gesonden / Maer selve heeft hi Lutherum beseten („Der Prinz der Teufel ist entfesselt / Und er hat seine Engel ausgesandt / Aber selbst hat er Luther besessen“).

15 Zu den religiösen Hintergründen des Bildersturms in Antwerpen: Van Eijnatten und Van Lieburg (2006); insbesondere zur Verbreitung des Calvinismus in Antwerpen und seine Rolle im Bildersturm: $163^{-165}$.

16 Ganz explizit verweist Pippinck am Ende seines Vorwortes auf die Ereignisse, wenn er berichtet, dass er seine Einleitung auf den Gedichtband schrieb in ons erm verbrant Cloos- 
nen neben der Bestätigung im Glauben für die katholische Leserschaft noch einen viel weltlicheren Zweck, nämlich das Sammeln von Geldern für den Wiederaufbau. Nicht umsonst lobte der Redakteur des Buches, der FranziskanerOrdensvorsteher Henrick Pippinck, in seiner Widmung die groote jonste ende [het] goet herte („die große Gunst und das gute Herz“) der Gräfin Maria von Mansfelt, die seinem Orden zuvor in Gelddingen wohl immer unter die Arme gegriffen hatte, und die hier ganz unverfroren um weitere Spenden angegangen wurde. Als Gegenleistung widmete er ihr das Buch als cleyn teeken der danckbaerheyt („kleines Zeichen der Dankbarkeit“). ${ }^{17}$

Alle drei Bände wurden im Laufe des sechzehnten Jahrhunderts und bis weit ins siebzehnte Jahrhundert hinein immer wieder nachgedruckt. Bijns refreinen erfreuten sich größter Beliebtheit beim niederländischen Publikum, auch lange nachdem Flandern nach dem Fall von Antwerpen in 1585 endgültig wieder katholisch geworden war. Bijns hatte sich als bedeutende Dichterin etabliert; ihre refreinen wurden sogar in einigen Städten als Schulbücher zugelassen. Zahlreiche refreinen findet man außerdem in anderen Quellen; immer wieder in Handschriften, aber auch in Drucken, die nicht nur als Dichtbände, sondern auch explizit als Liederbücher ihr Publikum finden sollten. Erst als der Geschmack sich grundlegend geändert hatte und die refreinen ab der Mitte des siebzehnten Jahrhunderts den Sonetten weichen mussten, geriet Bijns' Werk in Vergessenheit. ${ }^{18}$

ter der Minnebroeders inder stadt van Antwerpen (,in unserem armen verbrannten Kloster der Minderbrüder in der Stadt Antwerpen“; Keßler und Oosterman (2007c) 9). Scheerder zeigt, dass die Bilderstürmer die Ziele ihrer Aktionen ganz bewusst wählten. Sie hatten neben den Figuren und bildlichen Darstellungen und Kirchen auch ganz besonders die Klöster der Bettelorden im Auge, omdat de kloosterlingen inquisiteurs waren of in hun sermoenen de hervormden heftig bestreden hadden („weil die Mönche Inquisitoren waren oder in ihren Predigten die Reformierten heftig bekämpft hatten“; Scheerder (1978) 5758). Dass also gerade das Franziskanerkloster so stark verwüstet wurde, war demzufolge weder Zufall noch Willkür.

17 Über Pippinck: Van Heel (1940). Maria von Mansfelt stirbt am 5. Februar 1570, keine drei Jahre nach der Publikation des Buches. Zu ihr und ihrem Mann, Peter Ernst von Mansfelt: De Schepper (2009). Von Mansfelt ist ihr zweiter Ehemann nach Charles de Lalaing, der bereits $155^{8}$ starb. Übrigens gehört zum territorialen Besitz der Grafen von Mansfelt die Stadt Eisleben, die Geburtsstadt Martin Luthers; vgl. Geevers (2008) 139. Alle Zitate: Keßler und Oosterman $(2007 \mathrm{c}) 7$.

18 Zur Überlieferungsgeschichte: Die Drucke werden beschrieben in Roose (1963) 53-70. Die fünfzehn wichtigsten Handschriften beschreibt ebenfalls Roose (1963) 71-138; darin auch eine Beschreibung der Parallelüberlieferungen. Ebenso, aber wesentlich knapper:Van Elslander (1953) 21-23; Coigneau (1980-1983) Bd. 1, 73-109. Darüber hinaus zu Handschrift Brüssel, KB, II 270, der wahrscheinlich ältesten Quelle mit refreinen von Bijns: Lyna (1924); Willems (1925); Keßler (2007). 
Wenn wir uns nun insbesondere auf das refrein über den freien Willen richten, dann wird deutlich, dass dies ein Beispiel jener Gedichte von Bijns ist, wie sie vor allem in den ersten beiden gedruckten Sammelbänden zu finden sind: Es handelt sich um eines jener Gedichte, in denen sie voller Hass gegen Luther zu Felde zieht. Dabei verlässt sie sich in diesem Fall aber nicht auf Polemik, sondern sie versucht, auf der Basis theologischer Argumente ihre Leser zu überzeugen; dies ist das Ziel ihres Gedichtes. Sie argumentiert kontrovers, und zwar so, wie es dem lateinischen Wortsinn entspricht: contra versus, also gegen jemanden oder etwas gerichtet. Damit wird eine Gegenüberstellung zweier konträrer Standpunkte suggeriert. Und darum geht es auch: In Bijns' Denkmuster ist Luther ein Synonym für den Weg ins Verderben. Die katholische Tradition aber und ihre Interpretation vom Willen Gottes sind für Bijns ein Synonym für das absolut Gute. Dazwischen gibt es keinerlei Nuancen.

In diesem Spannungsfeld zwischen Gut und Böse schreibt Bijns ein Gedicht, das den freien Willen beweisen soll, und zwar aus den Worten der Heiligen Schrift. Das zeigt die kurze Einleitung, die dem Text vorangestellt ist. ${ }^{19}$ Dort wird gesagt, dies sei ein Refereyn declarerende uut der heyliger scriftueren / dat de menscen hebben eenen vryen wille („Refrein, das aus der Heiligen Schrift erklärt, dass die Menschen einen freien Willen haben“). Damit wird klar, wie Bijns ihre Argumentation aufbauen wird, nämlich immer ganz dicht am Bibeltext und gespickt mit zahlreichen Querverweisen zur Bibel. Dabei schreibt Bijns zwar gegen Luthers Auffassung, doch ohne ihm zu schreiben; es ist fraglich, ob Luther jemals auch nur ein refrein von ihr gelesen hat. Eine persönliche Kontroverse hat es wohl nicht gegeben. Im Gegenteil, Bijns schreibt für ihre Landsleute, für die (katholischen) Laien, denen deutlich gemacht werden soll, welcher Weg denn nun tatsächlich - Bijns' Meinung nach - zur Vergebung der Sünden führt. Sie sollen von der aus Bijns' Sicht verderblichen lutherischen Lehre ferngehalten werden.

Mit der Besprechung des refreins kommen wir zu der zentralen Frage nach dem Grund für die Wahl des Mediums in diesem speziellen Fall, in dem Bijns sich in die bestehende Diskussion rund um den freien Willen einbringt. Für die Wahl gibt es sowohl miteinander eng verbundene formale und inhaltliche Argumente als auch Argumente, die die im refrein angewandte Rhetorik betreffen.

19 Für den vollständigen niederländischen Text und eine deutsche Prosaübersetzung verweise ich auf den Anhang. 


\section{$3 \quad$ Form und Inhalt}

Zuvor habe ich erwähnt, dass Anna Bijns in diesem Gedicht zahlreiche Bibelzitate verarbeitet. Wenn man sich den Inhalt dieser Zitate ansieht, dann fällt auf, dass eigentlich alle Zitate dasselbe Thema besprechen, es aber in variierter Form wiedergeben. Als Beispiel sollen zwei Zitate aus der ersten Strophe dienen. Dort sagt Bijns zunächst in den Versen drei und vier:

Wildi tot mi keeren / ic keere mi tot dy Zacharie i

Dus roept god dicwils / so scriftueren leeren

Str. 1, V. 3-4

„Wenn Du Dich mir zuwendest, dann wende ich mich Dir zu“, / so sagt Gott oft, wie die Bibel lehrt.

Und weiter, zum Ende der Strophe:

Mijn avontmael is bereet naer tbetamen Luce xiiii

Die wilden quamen / god en heeft nyet gebroken

Comt tot mi al die dorst / roept Christus bi namen

Johan vii

Hebben wi geenen wille so ketters ramen

So sijn dees woorden te vergeefs gesproken.

Str. 1, V. 10-15

„Mein Abendmahl ist bereitet, wie es sich gehört / Diejenigen, die wollten, kamen. Gott hat sein Wort nicht gebrochen. / „Kommt alle zu mir, die dürstet", ruft Christus. / Wenn wir keinen freien Willen haben, wie die Ketzer behaupten, / dann wurden diese Worte umsonst gesprochen.

Die Bibelzitate, deren Quelle in diesem Gedicht immer auch in der Form von Marginalnoten angegeben werden (wie hier Zacharias 1, Lukas 14 und Johannes 7), haben einen sehr ähnlichen Inhalt. Immer wieder wird hier auf die Wahl angespielt, die Gott dem Menschen vorlegt: Gott hat alles vorbereitet, wie zum Beispiel das Abendmahl. Der Mensch muss nur noch frei-willig zu ihm kommen. Das impliziert ein Leben nach Gottes Geboten, aber um die genaue Durchführung desselben soll es in diesem Gedicht nicht weiter gehen. Wichtig ist für Bijns vielmehr, dass alle diese Bibelzitate beweisen sollen, dass Gott den Menschen immer wieder vor die Wahl stellt, und dass es des Menschen freie Entscheidung ist, Gottes Angebot anzunehmen. Dieses Angebot wird dem Publikum nun in allen fünf Strophen immer wieder und immer in leicht abge- 
änderter Form vorgelegt, wobei jedes Mal die Kernaussage - jeder Mensch hat einen freien Willen - in der stokregel (Vers 15) wiederholt wird. Durch die stete Variation auf dasselbe Thema mit derselben Technik (dem Zitieren aus der Bibel) scheint es von Strophe zu Strophe jedoch kaum eine inhaltliche Weiterentwicklung zu geben, im Gegenteil: Inhaltlich gesehen gibt es eigentlich kaum Gründe, die Strophen in genau dieser Reihenfolge stehen zu lassen. Es gibt nämlich keinerlei strophenübergreifenden inhaltlichen Verbindungen, außer eben der Tatsache, dass sie dasselbe Thema behandeln.

Die einzige ordnende Anweisung ist in der letzten Strophe zu finden, nämlich in ihrer Überschrift prince, die angibt, dass es sich hierbei um die letzte Strophe handeln muss. Da diese Strophe einen besonderen Status als Abschluss eines refreins hat, muss sie einen zusammenfassenden Charakter haben oder auf andere Weise den Höhepunkt des Gedichtes bilden. Das geschieht hier auch. In jeder Strophe werden ja die unendliche Gnade Gottes und seine Bereitschaft, dem Sünder zu verzeihen, anhand von Bibelzitaten bewiesen. Das wird in allen Strophen angedeutet und geschieht ganz explizit zum Beispiel in der prince-Strophe, weil dort die Schlussfolgerung aus dem Vorhergehenden gezogen wird. Dem Leser wird jetzt gesagt, was er denn eigentlich tun soll:

Werpt wech al u overdaet / doet mijn bevel

Maect u een nieu herte / laet u overspel

O huys van Israel / waer om suldi sterven

Ezec xviii

Seyt god duer den prophete Ezechiel

Ic en begeere sonders doot niet / al is hi fel

Str. 5 , V. $1-5$

„Werft alle Untaten von Euch, gehorcht meinem Befehl. / Macht Euch ein neues Herz, lasst Euren Betrug. / O Haus von Israel, warum solltest Du sterben?“ / So sagt Gott durch den Propheten Ezechiel. / "Ich will nicht, dass ein Sünder stirbt, auch wenn er böse war.“

Dass Gott wirklich gnädig ist, zeigt Bijns, indem sie den Wahrheitsgehalt der Aussage gleich doppelt betont; einmal nämlich, indem sie das Angebot zur Versöhnung und Vergebung als Worte Gottes bezeichnet, ihm die sogar in den Mund legt, und einmal, indem sie außerdem die Quelle dieser Worte expliziert: Ezechiel 18 (die genaue Stelle ist Kapitel 18, Vers 30). Es ist Gottes Wort also, das getreu in der Bibel niedergeschrieben wurde. An einer solchen Quelle darf nicht gezweifelt werden, das sollten sogar die Lutheraner einsehen, meint Bijns, die sich für alle theologischen Fragen ja ausschließlich auf die Schrift berufen. 
Es sind eben jene bibeltreuen Lutheraner, die insbesondere in der stokregel mit einem rhetorischen Kunstgriff vorgeführt werden. Syntaktisch gesehen handelt es sich bei dem stok um einen Halbsatz, der eine Folge angibt. Die dazugehörige Bedingung steht in einem Nebensatz, der noch zum vorherigen Vers gehört. Dabei wird die eigentliche Aussage des gesamten Gedichts ins Negative gekehrt. Bijns sagt nicht, wie man aufgrund der vielen Zitate erwarten könnte: "Jeder Mensch hat einen freien Willen“, sondern sie formuliert indirekt: „Wenn wir keinen freien Willen haben [...], dann wurden diese Worte umsonst gesprochen“. Mit „diese[n] Worte“ sind die Worte Gottes gemeint, in denen er den Menschen vor die Wahl zwischen Gut und Böse stellt, und die in der jeweiligen Strophe vorher mit zahlreichen Bibelzitaten belegt wurden. Dass diese Worte ausgesprochen wurden, ist also verbürgt und das müssen auch die Lutheraner annehmen. Wenn es sich bei diesen wiederholten Angeboten um Worte Gottes handelt, dann sind diese Angebote per definitionem wahr, denn an Gottes Wort ist nicht zu zweifeln. Wer dem Menschen den freien Willen, also die freie Wahl zwischen Gut und Böse verbietet, handelt somit gegen Gottes Willen und macht sich automatisch zum Ketzer. Damit ist diese Umkehrung, die Bijns jeweils in den letzten beiden Versen einer Strophe gebraucht, wesentlich effektvoller als die simple Feststellung, dass jeder Mensch einen freien Willen hat, denn dadurch kann Bijns nicht nur die katholische Bibellesung bestätigen, sondern gleichzeitig alle Andersdenkenden diffamieren.

Argumentationsstruktur

Die hiervor beschrieben Menge der Zitate und ihre Unterschiedlichkeit, oder auf Latein: die copia et varietas, ist seit der Antike ein bekanntes und gebräuchliches Stilmittel, um den Leser oder Zuhörer eines Textes zu überzeugen. Dazu kommt in diesem Fall als besonderes Überzeugungsmittel, dass die Argumentation im refrein sich auf die besondere Autorität der Bibelzitate stützt, da Gott oder Christus die Argumente selbst verwendet haben. Darum gibt Bijns zu allen Zitaten, die sie verwendet, an, von wem sie ursprünglich stammen. Und sie zitiert nicht nur, sondern sie lässt Gott und Christus in Figurenrede sogar selbst zu Wort kommen. ${ }^{20}$ Im gesamten Gedicht gibt es kein Zitat und somit kein

20 In der Übersetzung habe ich das kenntlich gemacht, indem ich an den betreffenden Stellen Anführungszeichen hinzugefügt habe. Grundlegend für das Studium der Rhetorik im Mittelalter sind immer noch die Studien von Murphy (1971, 1974 und 1983) sowie der Sammelband von Plett (1993). In meiner Untersuchung der Argumentationsstruktur folge ich jedoch den argumentationstheoretischen Ansätzen der „Amsterdamer Schule“, wie sie 
Argument, dass nicht mit einer Quellenangabe belegt wurde. Das zeigt sich am deutlichsten, wenn wir uns schematisch die argumentative Struktur des refreins vor Augen halten, hier am Beispiel der ersten Strophe:

These:

Die Menschen haben einen freien Willen; Gott überlässt dem Menschen die Wahl zwischen Gut und Böse

Argument 1: Gott will, dass die Menschen ein heiliges Leben führen Unterstützung 1: $\quad$ 1Thess $4,1^{21}$

Argument 2: $\quad$ Gott wird die Entscheidung für das Gute belohnen

Unterstützung 2: $\quad$ Zach 1, 3

Argument 3: $\quad$ Gott wird auch die beschützen, die gesündigt haben

Unterstützung 3: Jer 3,12-13

Argument 4: $\quad$ Gott sagt, er will die Menschen beschützen

Unterstützung 4: $\quad$ Matt 23,37

Argument 5: $\quad$ Gott möchte zusammen mit den Menschen das Abendmahl zu sich nehmen

Unterstützung 5: Luk 14, 12-24

Argument 6: $\quad$ Christus wird denjenigen zu trinken geben, die Durst haben

Unterstützung 6: Joh 7, 37-39

Wie aus diesem Schema hervorgeht, wird in der Argumentation in dieser Strophe (und das gilt auch für die übrigen vier Strophen) ausschließlich mit Bibelzitaten gearbeitet, die außerdem so korrekt wie möglich (also wie damals üblich mit Kapitelangaben) belegt werden. Bei diesen Zitaten fällt gleich auf, dass hier immer nur eine Seite der Entscheidung beleuchtet wird, und zwar indem die direkten Folgen einer Entscheidung des Menschen dargestellt werden. Wenn der Mensch sich für das Gute entscheidet, wird er von Gott belohnt werden, wird er beschützt werden, wird er zusammen mit Gott das Abendmahl einneh-

insbesondere von Schellens und Verhoeven (1994) propagiert wird. Ausführliche Informationen zur Methode in Keßler (2013).

21 Bijns verweist ausschließlich nach Kapiteln in den Büchern der Bibel. Die Versangaben habe ich hinzugefügt. 
men dürfen usw. Mit andere Worten: Wer das möchte, wer das Gute erreichen will, hat schlichtweg nur eine Möglichkeit, sich zu entscheiden. Genauso wird in anderen Strophen das Schlechte gezeigt, das dann von Gott bestraft werden wird. Niemals jedoch wird Bijns sagen, dass eine Entscheidung gegen die katholische Auslegung von Gottes Wort - und damit gegen das Gute - positive Folgen haben kann, weil das schlichtweg für sie undenkbar ist. Auch wenn der Mensch, wie sie hier sagt, einen freien Willen hat: Die Entscheidung, die er „freiwillig“ nehmen soll, zeichnet sie ihm ganz genau vor, indem sie die möglichen Folgen erörtert. Solchen Argumenten kann einfach nicht widersprochen werden - zumindest dann, wenn man sich der katholischen Interpretation anschließen kann. Wieder zeigt sich, dass das refrein sich nur an ein katholisches Publikum richten kann, das sowieso schon Bijns' Sicht der Dinge weitgehend zustimmt.

\section{5}

\section{Erfolge}

Die Struktur einer jeden Strophe ist extrem einfach und gleichmäßig, und durch die inhaltliche Ähnlichkeit mit der besonderen Betonung der wiederkehrenden stokregel ist das gesamte refrein zyklisch, um nicht zu sagen: redundant. Bijns wirbt damit fast schon aufdringlich für das Gute. Wie können Gedichte wie dieses, das sich nicht an die klassischen Regeln guter Rhetorik hält und das keinen geradlinigen Aufbau kennt, so erfolgreich sein, dass Bijns die Technik regelmäßig anwendet und gleich drei Gedichtbände damit füllen kann? Darüber hinaus handelt es sich um Gedichtbände, die mehrfach neu aufgelegt werden und die bis ins siebzehnte Jahrhundert hinein begeistert gelesen werden. Was also macht die Gattung des refreins so geeignet, um eine große Leserschaft zu erreichen? Anders gefragt: Gab es Alternativen?

Die Antwort lautet schlicht: Nein, es gab keine Alternativen. Jedenfalls gab es die nicht für Bijns als Frau, die allein wegen ihres Geschlechts weitaus weniger Möglichkeiten für eine schulische oder gar universitäre Ausbildung hatte als Männer; sie hatte natürlich auch keinen Zugang zum Priestertum. Insofern muss ihr der Weg zu den typischen Gattungen gelehrter Konversation von vorneherein verschlossen gewesen sein. Doch man ist sich einig, dass Bijns trotz ihrer wohl kurzen Schullaufbahn eine gebildete Frau war. Gerade das refrein über den freien Willen zeigt, dass sie sowohl das Thema als auch die Diskussion darüber (und die geeigneten Bibelstellen) sehr genau kannte.

Zu der Zeit, als Anna Bijns in der Kontroverse Stellung bezog, war das refrein bei den rederijkers und beim Publikum gleichermaßen beliebt. Diese Gattung 
war durchweg volkssprachig, denn sowohl bei den rederijkers als bei ihrem Publikum handelte es sich um Bürger, die nur wenig oder kein Latein konnten. Ein refrein war also für alle problemlos zu verstehen, denn es war auf sein Publikum zugeschnitten. Hier schrieben theologische Laien für ein Publikum auf Augenhöhe, das eine Erwartungshaltung in Bezug auf Literatur aufgebaut hatte, auf Formen und Inhalte, auf Rhythmus und Reim, auf den Unterhaltungswert durch die ästhetische Form und auf die Ernsthaftigkeit, mit der inhaltlich eine Meinung vorgebracht wurde. Vor diesem Hintergrund blieb Bijns, wenn sie ein größeres Publikum erreichen wollte, nur die Möglichkeit, so zu dichten, wie die populären rederijkers es taten: im refrein.

Aber das refrein bietet auch für den Autor bzw. die Autorin allerlei reizvolle Möglichkeiten literarischer Art. Hier kann er sein ganzes Können zeigen, zum Beispiel was den Reim angeht, was Sprach- und Wortspiele betrifft und die Kunst, einen komplexen theologischen Sachverhalt in relativ wenigen, relativ einfachen Worten auszudrücken, und dabei noch anspruchsvollen formalen Regeln Genüge zu tun. Genau das zeichnet die refreinen von Bijns aus: die komplizierte Frage nach dem freien Willen wird mittels einfach zu verstehender und vermutlich sehr bekannter Bibelzitate auf ein für kirchliche Laien verständliches Niveau herunter gebrochen. Die Laien können jetzt mitdiskutieren und sich der Meinung von Bijns anschließen (oder eben nicht). Die ansprechende Form des refreins mit seinem Rhythmus und seinem Reim tut ein Übriges um die eingängige Botschaft im Gedächtnis des Publikums zu verankern, die durch die ständige Wiederholung in den fünf Strophen zudem immer tiefer eingegraben wird.

Vielleicht ist das auch der Grund des Verkaufserfolges insbesondere des ersten Gedichtbandes von Bijns, der insgesamt fünfmal gedruckt wurde: Dass sie mit dem Balanceakt aus Form und Inhalt meisterlich umzugehen verstand, und dass sie ein breites Publikum mit ihrem Werk erreichen konnte. Indem Bijns das Thema allgemein verständlich macht, gibt sie den Zuhörern und Lesern die Gelegenheit, sich über die Debatte zum freien Willen zu informieren und sich darüber eine Meinung zu bilden. Dass diese Meinung vorgeformt wurde und höchst einseitig ist, spielt erst einmal eine Nebenrolle. Die Gegenseite wird nämlich von den zahlreichen lutherischen refreinen vertreten, die gleichzeitig im Umlauf waren und auf die Bijns vor allem in ihrem zweiten Band wiederholt (und noch viel schärfer als im ersten Band) reagiert. Viel wichtiger ist, dass Bijns den Leser dort abholt, wo er mit seinem Wissen steht. Ihr refrein ist ganz und gar eine Einladung, sich ihrer Meinung anzuschließen, und die feste Verquickung zwischen Form, Inhalt und Argumentationsstruktur macht es einfach, genau das zu tun. 
Es ist eine komplexe Mischung von Gründen, die in Bijns' Fall für den Gebrauch des refreins als zu bevorzugende Form für ihre Botschaft sprechen. Dabei zeigt sich, dass hier keineswegs nur inhaltliche und formelle Gründe eine wichtige Rolle spielen, sondern dass auch die Erwartungshaltung des Publikums nicht unterschätzt werden darf. Die rederijkers haben bis zu diesem Zeitpunkt eine so weit reichende und einflussreiche Gattungstradition geschaffen, dass ein Autor, der sein Publikum in der Volkssprache erreichen will, kaum eine andere Wahl hat als sich an diese Tradition zu halten. Gerade die Gedichte von Bijns zeigen dann, wie gut es ihr gelingt, erstens den literarischen Ansprüchen der rederijkers zu genügen, zweitens das Publikum zu erreichen und drittens ihren Standpunkt in der konfessionellen Kontroverse zu behaupten.

Und letztlich: Liegt es nicht eigentlich in der Verantwortung der Lutheraner, wenn Bijns gegen sie schreibt, und zwar in refreinen? Sie lässt uns in einem kleinen Gedicht kurz vor Ende des des zweiten Gedichtband (aus circa 1548) Folgendes wissen:

Al hebic hier gescreven wat verwytelijc // yet Tegen de Luteranen ten es gheen wonder // vry Want sy scryven wel noch eens so spytelijc // siet Teghen de heylighe kercke daer sy tonder // by Brenghen tgheloove ic fundere bysonder // my Opt woort dat de Wijseman sonder spot // seyt Ghy seldt den sot antwoorde naer sijn sotheyt

Damit hat Bijns ihr ganzes Tun gerechtfertigt. Denn die Lutheraner sind Narren, denen man mit gleicher Münze heimzahlen soll; so jedenfalls schreibt es die Bibel vor. Deshalb darf Bijns genauso frech und polemisch schreiben wie die Lutheraner es tun - nicht, um ihnen gleich zu werden, sondern damit sie nicht denken, dass sie weise seien (Sprichwörter 26, 4-5). Mehrmals betont sie in diesem zweiten Band, dass sie gegen lutherische refreinen schreibt. Diese Bibelstelle ist also sowohl ein Argument für das Schreiben gegen Luther als auch für das Schreiben in refreinen, denn die Lutheraner haben ja mit allem angefangen.

22 Wenn ich hier etwas gegen die Lutheraner geschrieben habe, das man mir übel nehmen könnte, dann ist das kein Wunder. Denn sie schreiben mindestens so böse gegen die heilige Kirche, deren Glaube sie untergraben. Ich stütze mich hier insbesondere auf das Wort, das der Weise Mann [im Buch der Sprichwörter] ohne Spott sagt: „Antworte dem Narren nach seiner Narrheit". 
Das lässt sich zu einem poetologischen Gegensatz zur inhaltlichen Kontroverse zuspitzen: Man ist sich einig im Medium, aber man verfolgt zweierlei Meinungen im Inhalt. Es gibt hier also offenbar keine konfessionell geschiedene Poetik der lyrischen Kontroverse, denn das Mittel als solches hat sich bewährt, aber die inhaltlichen Ziele dieser sich einmischenden Lyrik könnten unterschiedlicher kaum sein.

\section{Literatur}

Akerboom, T.H.M. 1995. Vrije wil en/of genade. Een theologie-historisch onderzoek naar het dispuut tussen Erasmus en Luther over de (on)vrijheid van het menselijke willen. Amsterdam.

Akerboom, D., und Gielis, M. 2005. „A New Song Shall Begin Here ..... The Martyrdom of Luther's Followers among Antwerp's Augustinians on July 1, 1523 and Luther's Response. In More than a Memory. The Discourse of Martyrdom and the Construction of Christian Identity in the History of Christianity, hgg. J. Leemans. Leuven: 243-270.

Bautz, F.W. 2008. Biographisch-bibliographisches Kirchenlexikon. Bd. XIII. Nordhausen (Online-Edition: http://www.bautz.de/bbkl/, Version vom 5. September 2013).

Bayer, O. 2003. Martin Luthers Theologie. Eine Vergegenwärtigung. Tübingen.

Bogaers, A., und Van Helten, W.L. (Hg.) 1875. Anna Bijns, Refereinen van Anna Bijns. Naar de nalatenschap van Mr. A. Bogaers. Rotterdam.

Van den Branden, F.J. 1911. Anna Bijns. Haar leven, hare werken, haar tijd. 1493-1575. Antwerpen.

Van Bruaene, A.-L. 2008. Om beters wille: Rederijkerskamers en de stedelijke cultuur in de Zuidelijke Nederlanden (1400-1650). Amsterdam.

Van Bruaene, A.-L. 2004. Repertorium van rederijkerskamers in de Zuidelijke Nederlanden en Luik 1400-1650. Online-Edition DBNL (http://www.dbnl.nl/tekst/bruaoo2rep eo1_o1/index.htm).

Coigneau, D. 1980-1983. Refreinen in het zotte bij de rederijkers. 3 Bde. Gent. (Koninklijke Vlaamse Academie voor Taal- en Letterkunde, vi. Reihe. Nr. 111).

Van Dixhoorn, A. 2004. Lustige geesten. Rederijkers en hun kamers in het publieke leven van de Noordelijke Nederlanden in de vijftiende, zestiende en zeventiende eeuw. Amsterdam.

Van Eijnatten, J., und Van Lieburg, F. 20o6. Nederlandse religiegeschiedenis. 2. Auflage. Hilversum. Online-Edition (http://books.google.nl/books?id=noOPgJYg18QC, Version 5. September 2013).

Van Elslander, A. 1953. Het refrein in de Nederlanden tot 1600. Gent (Koninklijke Vlaamse Academie voor Taal- en Letterkunde, Reihe VI, Nr. 71).

Erasmus, D. 1524. De libero arbitriodiatribe sive collatio. Wien. Online-Edition (http:// 
books.google.de/books?id=9LQ8AAAAcAAJ\&pg=PT72\&dq=inauthor:Desiderius $+\mathrm{i}$ nauthor:Erasmus+libero+arbitrio\&lr=\&as_brr $=1 \# \mathrm{v}=$ onepage $\& \mathrm{q}=\& \mathrm{f}=\mathrm{false}$, Version 5. September 2013).

Folkers, H. 2006. Martin Luther: Vom unfreien Willen und der Freiheit eines Christenmenschen. Atem und Verantwortlichkeit in Luthers Denken. Kerygma und Dogma. Zeitschrift für theologische Forschung und kirchliche Lehre 52: 288-302.

Geevers, E. 2008. Gevallen vazallen. De integratie van Oranje, Egmont en Horn in de Spaans-Habsburgse monarchie (1559-1567). Amsterdam.

Geirnaert, D. (Hg.) 1986. Matthijs de Castlein, De const van rethoriken. Faksimile der ersten Ausgabe (Gent 1555). Oudenaarde. Online-Edition (http://www.dbnl.org/tekst/ castoo5conso1_o1/, Version vom 25. April 2007).

Van Heel, D. 1940. De minderbroeder Henricus Pippinck. Franciscaansch Leven. Maandschrift voor Franciscaansche Ascetiek, Geschiedenis en Kunst 23: 44-57.

Hummelen, W. 1958. De sinnekens in het rederijkersdrama. Groningen.

Janssen, H.Q. 1862. Jacobus Praepositus, Luthers leerling en vriend. Geschetst in zijn lijden en strijden voor de Hervormingszaak. Amsterdam.

Jonckbloet, W.J.A., und Van Helten, W.L. (Hg.) 1886. Anna Bijns, Nieuwe refereinen van Anna Bijns benevens enkele andere rederijkersgedichten uit de XVIe eeuw. Gent.

Keßler, J. 2007. Wie is Cornelis Damasz? Nieuws over handschrift Brussel, кB, II 270. Nederlandse Letterkunde 12: 94-117.

Keßler, J. 2013. Princesse der rederijkers. Het oeuvre van Anna Bijns: argumentatieanalyse - structuuranalyse - beeldvorming. Hilversum (Middeleeuwse studies en bronnen 145).

Keßler, J., und Oosterman, J. (Hg.) 2007a. Anna Bijns: Schoon ende suverlijc boecxken inhoudende veel constige refereinen. Online-Edition DBNL (http://www.dbnl.nl/ tekst/bijnoo3refeo1_o1/index.htm).

Keßler, J., und Oosterman, J. (Hg.) 20o7b. Anna Bijns: Tweede boeck vol schoone ende constighe refereynen. Online-Edition DBNL (http://www.dbnl.nl/tekst/bijnoo3refeo 2_o1/index.htm).

Keßler, J., und Oosterman, J. (Hg.) 2007c. Anna Bijns, Seer scoon ende suyver boeck, verclarende die mogentheyt Gods, ende Christus ghenade, over die sondighe menschen. Online-Edition DBNL (http://www.dbnl.nl/tekst/bijnoo3refeo3_o1/index.htm).

Lohse, E. 2006. Martin Luther und der Römerbrief des Apostels Paulus. Biblische Entdeckungen. Kerygma und Dogma. Zeitschrift für theologische Forschung und kirchliche Lehre 52:106-125.

Luther, M. 1526. Das der freie Wille nichts sey. Wittenberg 1526. Übersetzt von Justus Jonas. Online-Edition (http://daten.digitale-sammlungen.de/bsbooo27194/image_1, Version 5. September 2013).

Lyna, F. 1924. Een teruggevonden handschrift (Brussel, Hs. II 270). Tijdschriftvoor Nederlandse Taal- en Letterkunde 43: 289-323. 
Moser, N. 2001. De strijd voor rhetorica. Poëtica en positie van rederijkers in Vlaanderen, Brabant, Zeeland en Holland tussen 1450 en 1620 . Amsterdam.

Murphy, J.J. 1971. Three Medieval Rhetorical Arts. Berkeley, Los Angeles/London.

Murphy, J.J. 1974. Rhetoric in the Middle Ages. A History of Rhetorical Theory from Saint Augustine to the Renaissance. Berkeley, Los Angeles/London.

Murphy, J.J. (Hg.) 1983. Renaissance eloquence. Studies in the Theory and Practice of Renaissance Rhetoric. Berkeley, Los Angeles/London.

Oosterman, J., und Ramakers, B. (Hg.) 2001. Kamers, kunst en competitie. Teksten en documenten uit de rederijkerstijd. Amsterdam.

Pleij, H. 200o. Anna Bijns als pamflettiste? Het refrein over de beide Maartens. Spiegel der Letteren 42: 187-225.

Pleij, H. 2007. Het gevleugelde woord. Geschiedenis van de Nederlandse literatuur 14001650. Amsterdam (Geschiedenis van de Nederlandse literatuur, Bd. 2).

Pleij, H. 2011. Anna Bijns, van Antwerpen. Amsterdam.

Plett, H.F. (Hg.) 1993. Renaissance-Rhetorik. Renaissance Rhetoric. Berlin.

Ramakers, B. (Hg.) 2003. Conformisten en rebellen. Rederijkerscultuur in de Nederlanden (1400-1650). Amsterdam.

Robson, M. 2006. The Franciscans in the Middle Ages. Woodbridge (Monastic Orders).

Roest, B. 2000. A history of Franciscan education (ca. 1210-1517). Leiden (Education and society in the Middle Ages and Renaissance, vol. 11).

Roose, L. 1963. Anna Bijns. Een rederijkster uit de hervormingstijd. Gent.

Scheerder, J. 1978. De beeldenstorm. Haarlem. Online-Dokument (http://www.theologie net.nl/documenten/Scheerder-Beeldenstorm.pdf; Version 2008).

Schellens, P.J., und Verhoeven, G. 1994. Argument en tegenargument. Analyse van betogende teksten. 2de, herziene druk. Groningen.

De Schepper, H. 20o9. Peter Ernst, graaf van Mansfeld. Online-Dokument (http://www .dutchrevolt.leiden.edu/dutch/personen/M/Pages/Mansfeld_Peter_Ernst.aspx, Version 9. September 2013).

Schlager, P. 1904. Beiträge zur Geschichte der Kölnischen Franziskaner-Ordensprovinz im Mittelalter. Köln.

Schlager, P. 19o9. Geschichte der kölnischen Franziskaner-Ordensprovinz während des Reformationszeitalters. Regensburg.

Schneiderwirth, M. 1933. Anna Bïns. Eine flämische Lehrerin und Dichterin des 16. Jahrhunderts. Paderborn.

Soens, E. (Hg.) 1902. Onuitgegeven gedichten van Anna Bijns. Leuvensche Bijdragen 4: 199-368.

Waite, G.K. 2003. On the stage and in the street. Rhetorician drama, social conflict and religious upheaval in Amsterdam (1520-1566). In Conformisten en rebellen. Rederijkerscultuur in de Nederlanden (1400-1650), hgg. B. Ramakers. Amsterdam: 162173 . 
Wenz, G. 1998. Desiderius Erasmus: De libero arbitrio (hg. nach der Übersetzung von Otto Schumacher). Göttingen.

Willems, L. 1925. Over gedichten van Anth. de Roovere, van Hamme, van Frans Oisstoc, van Anna Byns enz. voorkomende in den Brusselschen Codex II, 27o. Verslagen en Mededelingen der Koninklijke Vlaamsche Academie voor Taal- en Letterkunde 1925: $832-839$.

\section{Anhang: So sijn dees woorden te vergeefs gesproken (mit Prosaübersetzung)}

Refereyn declarerende uut der heyliger scriftueren / dat de menscen hebben eenen vryen wille.

ons heylicheyt is / den wille ons heeren

Thessa iiii

Dat wy souden laten / duechden vermeeren

Wildi tot mi keeren / ic keere mi tot dy

Zacharie i

Dus roept god dicwils / so scriftueren leeren

Al hebdi u van veel boels laten onteeren

Wilt niet verweeren / maer keert weder tot mi

Hiere iii

Hierusalem / Hierusalem / hoe comet bi

Ic heb u seyt hi / dicwils willen versamen

Mat xxiii

Gelijc een hinne / haer kiecxkens onder mi vry

Ghi en wilt niet tfij / ghi muecht $\mathrm{u}$ wel scamen

Mijn avontmael is bereet naer tbetamen

Luce xiiii

Die wilden quamen / god en heeft nyet gebroken

Comt tot mi al die dorst / roept Christus bi namen

Johan vii

Hebben wi geenen wille so ketters ramen

So sijn dees woorden te vergeefs gesproken

Refrein, das aus der Heiligen Schrift erklärt, dass die Menschen einen freien Willen haben.

Dass wir ein heiliges Leben führen und dass wir die Tugend immer weiter vermehren, ist der Wille unseres Herrn. „Wenn du dich zu mir hinwendest, dann wende ich mich Dir zu“, so sagt Gott oft, wie die Bibel lehrt. „Auch wenn Du Dich durch viele schlechte Dinge hast entehren lassen, wehre Dich nicht, sondern wende dich mir zu. Jerusalem, Jerusalem, wie kann es so weit kommen. Ich habe Dich“, sagt er, „oftmals unter mir versammeln wollen, wie eine Henne es mit ihren Küken macht." Du willst nicht, pfui, Du müsstest Dich schämen. „Mein Abendmahl ist bereitet, wie es sich gehört.“ Diejenigen, 
die wollten, kamen. Gott hat sein Wort nicht gebrochen. „Kommt alle zu mir, die dürstet“, ruft Christus. Wenn wir keinen freien Willen haben, wie die Ketzer behaupten, dann wurden diese Worte umsonst gesprochen.

God seyt / in Deuteromio eest bescreven

Siet ic legge u te voren dees woorden cleven

Deute xi

Deewich leven / oft de vermaledidinge

Laet ghi mijn geboden ghi wert verdreven

Eest dat ghise hout / eewelic verheven

U wert gegeven / de hoochste verblidingen

Leest Joannem in deerste sonder vermidinge

Johan i

Hoort sijn belidinge / god geeft ons macht

Kinderen gods te werden / noch seker tijdinghe

Na ons besnidinge / staet Cristus en wacht

Voor tduerken en clopt / esser niemant wel bedacht

Apoca iii

Die mijn stemme acht / is de duere ontploken

Ic sal mijn avontmael daer houden dach ende nacht

Dit seyt god / is dan den vryen wille versmacht

So sijn dees woorden te vergeefs gesproken

Gott sagt, wie in Deuteronomium beschrieben steht: „Sieh, was ich Dir vorlege. Mit diesen Worten ist das ewige Leben verbunden oder die Verdammnis. Hältst Du Dich nicht an meine Gebote, wirst Du vertrieben werden. Hältst Du Dich daran, dann werden Dir die höchsten Freuden gegeben werden." Lies Johannes im ersten Kapitel, höre, was er bezeugt: „Gott gibt uns noch eine bestimmte Zeit lang die Macht, Kinder Gottes zu werden. Nach unserer Beschneidung steht Christus vor der Türe und klopft an, „wenn niemand damit rechnet meine Stimme zu hören. Wird die Türe geöffnet, dann werde ich dort Tag und Nacht mein Abendmahl halten." Das sagt Gott. Ist also der freie Wille verschmachtet, dann wurden diese Worte umsonst gesprochen.

Esser geenen vryen wille / liecht Christus dan

Die seit / wie na mi wilt comen tsi wijf oft man

Matheus scrijft hier van / ten sijn / geen sagen

Mat xvi

Wat sprac god tot Caym / eer hi de moort began

Ghi mueget tquaet verwinnen / dwelc hi niet en verwan

Maer heeft nochtan / sinen broeder verslaghen

Gene iiii

Twas sijn scult / niet gods scult quam hy in plagen

Hoort Salomons gewagen / die de waerheyt vant

Eccle xvii

Voor u staet water en vier / wilt niet clagen

Kiest na u behagen / mijn siel is in mijn hant 
Seyt David verlicht metten godliken brant

Ontbint desen bant / zijn u oogen geloken

Ghi die errueren stroyt / over alle dlant

Esser geenen vryen wille / na u erch verstant

So sijn dees woorden te vergeefs gesproken

Gibt es keinen freien Willen, lügt Christus also? Er sagt: „Komm zu mir, ob Du Frau oder Mann bist.“ Matthäus schreibt darüber, es sind keine Märchen. Was sprach Gott zu Kain, bevor der den Mord beging? „Du sollst das Böse überwinden“, was er nicht überwunden hat, denn er hat trotzdem seinen Bruder erschlagen. Es war seine Schuld, nicht Gottes Schuld, wenn er mit Plagen gestraft wurde. Hört, was Salomo spricht, der die Wahrheit erkannte: „Vor Dir stehen Wasser und Feuer. Klage nicht. Wähle, was Du willst.“ „Meine Seele liegt in meiner Hand“, sagte David, der vom göttlichen Feuer erleuchtet war. Entferne dieses Band, wenn Deine Augen geschlossen sind, der Du im ganzen Land Irrlehren verbreitest. Gibt es keinen freien Willen, Deinem bösen Verstand zufolge, dann wurden diese Worte umsonst gesprochen.

Ons gesontmaker Christus noch openbaer seyt

Mat xi

Comt alle tot mi ghi die daer arbeyt

Uwen last / u swaerheyt / sal ic u verlichten

Mat vii

Gaet duer dinge poorte / die int leven hier na leyt

Joan xii

Die wile dat ghi dlicht hebt / wandelt inde claerheit

Seyt de ewige waerheit / verstaet mijn dichten

Paulus scrijft / tot ons sondige wichten

Wilt seyt hi op richten / u slappe knien

Hebre xii

Doet duecht sonder ophouden / en wilt niet swichten

Voor sviants scichten / leert scriftuere ons vlien

Hout de geboden / wildi deewich leven sien

Seit Cristus van wyen / wert alle quaet gewroken

Mat xix

Tes gods wille / dat salich werden alle lien

Hebben wi geenen vryen wille ic blijf bi dien

i Timo ii

So sijn dees woorden te vergeefs gesproken

Unser Gesundmacher Christus sagte noch öffentlich: „Kommt alle zu mir, die Ihr arbeitet. Eure schwere Last will ich erleichtern. Geht durch die schmale Pforte, die in das Leben nach diesem Leben führt. Wenn Ihr das Licht habt, wandelt Ihr in der Helligkeit.“ Das sagt die ewige Wahrheit, versteht, was mein Gedicht sagen will. Paulus schreibt an uns sündige Wichte: „Wollt Ihr“, so sagt er, „Eure schlaffen Knie aufrichten. Verhaltet Euch immerzu tugendhaft und brecht nicht zusammen unter der Last. Vor den Fallen des Feindes lehrt uns die Bibel zu fliehen. Haltet Euch an die Gebote, wenn Ihr das 
Ewige Leben sehen wollt." So sagt Christus, durch den alles Böse gerächt wurde. Es ist Gottes Wille, dass alle Menschen selig werden. Ich bleibe dabei: wenn wir keinen freien Willen haben, dann wurden diese Worte umsonst gesprochen.

Prince.

Werpt wech al u overdaet / doet mijn bevel

Maect $u$ een nieu herte / laet $u$ overspel

O huys van Israel / waer om suldi sterven

Ezec xviii

Seyt god duer den prophete Ezechiel

Ic en begeere sonders doot niet / al is hi fel

Maer keert hi hem snel / so menich werven

Ezechi xviii

Als hi sucht voor sijn sonden / sal icse af kerven

En hi sal erven / mijn rijcke reene

Gods graci es bereet / soet boven conserven

Dat wi dan bederven / tis ons scult alleene

Keert weder ende leeft / seit god tot groot en cleene

Titum ii

Ic en verstoot geene / comt onder mi gedoken

Mat iii

Doet penitentie roept hi al ghemeene

Hebben wi geenen wille / elc trect te beene

So sijn dees woorden te vergeefs gesproken

Prinz.

„Werft alle Untaten von Euch, gehorcht meinem Befehl. Macht Euch ein neues Herz, lasst Euren Betrug. O Haus von Israel, warum solltest Du sterben?“ So sagt Gott durch den Propheten Ezechiel. „Ich will nicht, dass ein Sünder stirbt, auch wenn er böse war. Aber, oftmals, wenn er schnell umkehrt, wenn er seine Sünden bereut, dann werde ich sie vergeben und er wird mein reines Reich erben." Gottes Gnade ist bereit, sie ist süß wie Konserven. Dass wir es verderben, ist allein unsere Schuld. „Kehrt um und lebt“, sagt Gott zu groß und klein. „Ich verstoße niemanden. Duckt Euch unter mir. Tut Buße“, ruft er allen zu. Merkt auf:Wenn wir keinen freien Willen haben, dann wurden diese Worte umsonst gesprochen. 